\title{
A SEARCH FOR FEATURES IN EARLY-TYPE GALAXIES
}

S. Djorgovski and K. Ebneter

Astronomy Department

University of California

Berkeley, CA 94720

U.S.A.

ABSTRACT. We are conducting a search for dust lanes, incipient stellar disks and bars, and other deviations from elliptical symmetry in a sample 250 early-type galaxies. Division by a model galaxy image is a very powerful enhancement technique for this type of work.

A classical image of elliptical galaxies contains no dust or stellar disks: these should be the attributes of spirals. Modern data do not support this simple picture. Many bona fide ellipticals are now known to contain dust (cf. Ebneter \& Balick 1985, or Salder \& Gerhard 1985). The presence of dust in elliptical galaxies is most likely a signature of of past mergers, and it provides some clues about the origin of nuclear activity in galaxies. The morphological and kinematical separation of ellipticals and SO's is getting more and more blurred. A continuity of galaxian properties between different morphological types is of great importance for theories of galaxy formation and evolution.

We are currently completing a large search for morphological features in early-type galaxies, viz., all deviations from the elliptical symmetry of the underlying light. The data come from the CCD surface photometry by Djorgovski (1985), and cover 200 elliptical and $\sim 50$ so galaxies. This work is fairly similar to the surveys of Lauer (1985), and Sparks et al. (1985), and employs the same digital processing technique, which we describe below. The difference is primarily in the sample size, as we are covering several times more objects than the previous investigators; the sample selection effects are well under control.

The main problem here is essentially the suppression of the strong radial intensity gradient. This can be accomplished by a polar-coordinates gradient technique (radial stretch or squeeze, possibly a slight rotation, and subtraction from the original image). We tried that method, and found it satisfactory, but another technique worked better, viz., division by the purely elliptical galaxy model image. The method works as follows:

From the surface photometry of a galaxy (best fit surface brightness, ellipticity, and position angle as functions of semimajor axis), we reconstruct a model galaxy image which obeys those profiles, but has 
purely elliptical isophotes; it helps to smooth the $\varepsilon$ and PA profiles somewhat. The model is smoother than the original data frame, because of the isophotal averaging, and smoothing of the $\varepsilon$ and PA profiles. We then divide the original image by the smoothed image. The reason for superiority of this technique over the simple polar gradient is that we are adding some a priori information in the image restoration process, viz., the exact form of the galaxy light distribution, plus the demand that the "unperturbed" galaxy has purely elliptical isophotes.

\section{REFERENGES:}

Djorgovski, S. 1985, Ph. D. Thesis, University of California at Berkeley. Ebneter, K., and Balick, B. 1985, Astron. J. 90, 183.

Lauer, T.R. 1985, M.N.R.A.S. 216, 429.

Sadler, E.M., and Gerhard, O.E. 1985, M.N.R.A.S. 214, 177.

Sparks, W.B. et al.1985, M.N.R.A.S. 217, 87 .

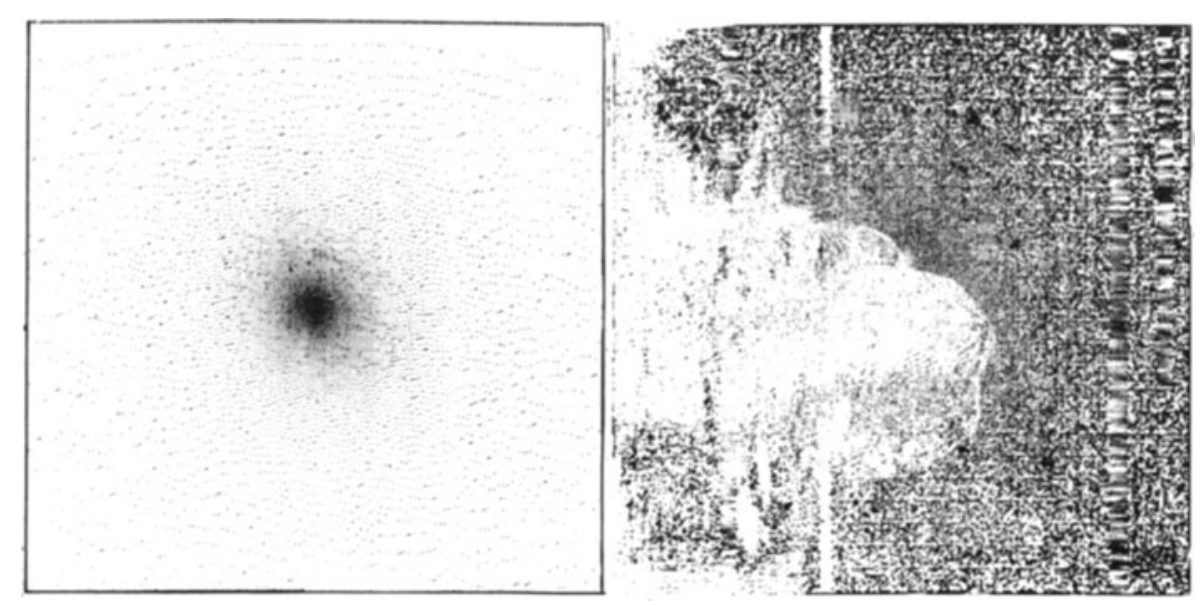

Figure 1: An example of feature enhancement. The original image is on the left and the model-divided image is on the right. The object is NGC4374 which reveals a dust lane (a ring?); note the limb-darkening of the ring: the dust is optically thin! 


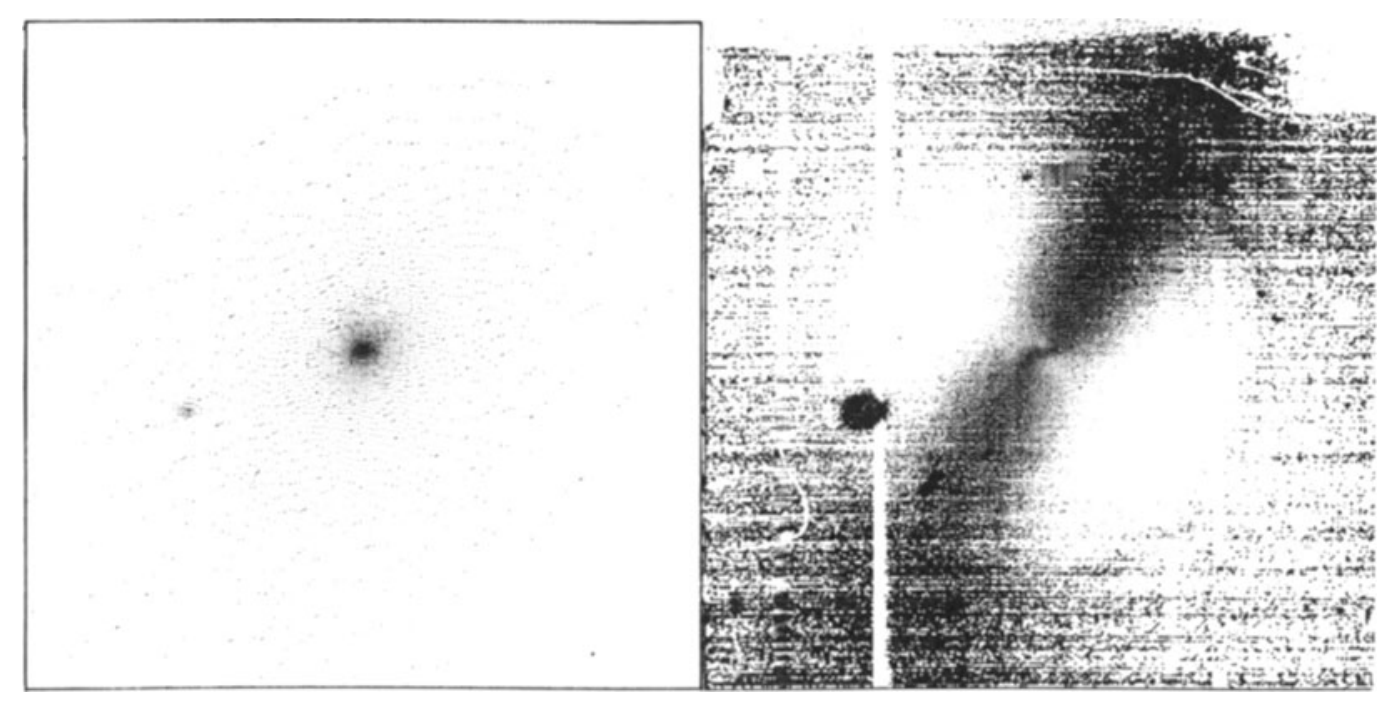

Figure 2: Another example of feature enhancement, this time for NGC 584 which shows a thick stellar disk, and a smaller, tilted one in the nucleus. 